\title{
Improved k-space-based MR thermometry by joint PRF phase shift and $\mathrm{T}_{1 / \mathrm{T}}{ }^{*}$ attenuation estimation
}

\author{
Pooja Gaur*, William Grissom
}

From Current and Future Applications of Focused Ultrasound 2014. 4th International Symposium Washington, D.C, USA. 12-16 October 2014

\section{Background/introduction}

MR temperature mapping based on the proton resonance frequency (PRF) shift is used in MR-guided focused ultrasound procedures for dosimetry and safety monitoring. While conventional PRF-shift thermometry is based on calculating a phase difference between two reconstructed MR images, Gaur et al [1,2] have recently described two algorithms that estimate temperatureinduced phase shifts directly from MR k-space data, prior to image reconstruction. The approach enables

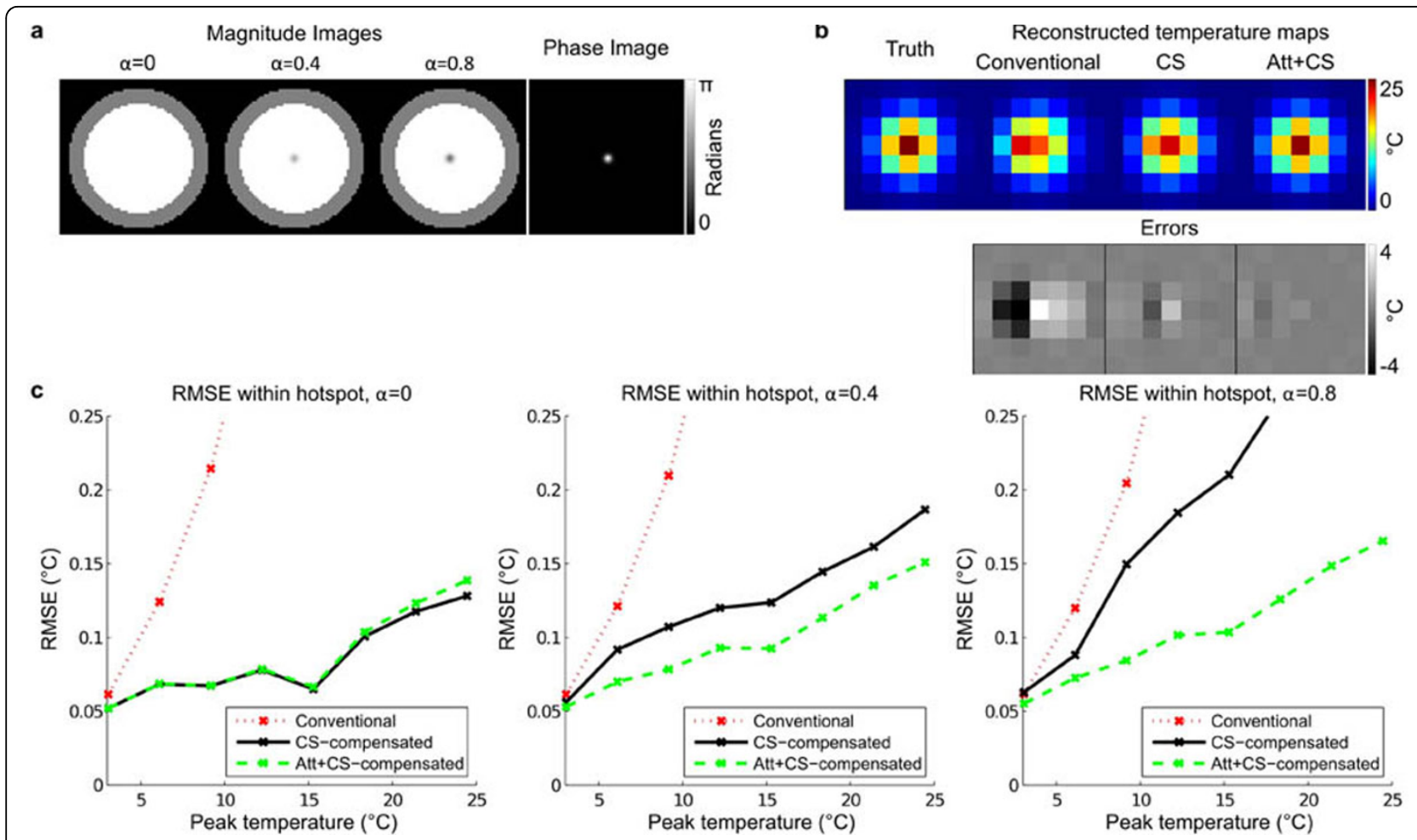

Figure 1 Simulation results. (a) Magnitude images with attenuation factors (alpha) and phase image. (b) Comparison of true and reconstructed temperature maps using the three algorithms. (c) Root-mean-square-error (RMSE) over the hot spot at 3 attenuation factors.

Vanderbilt University, Nashville, Tennessee, United States 


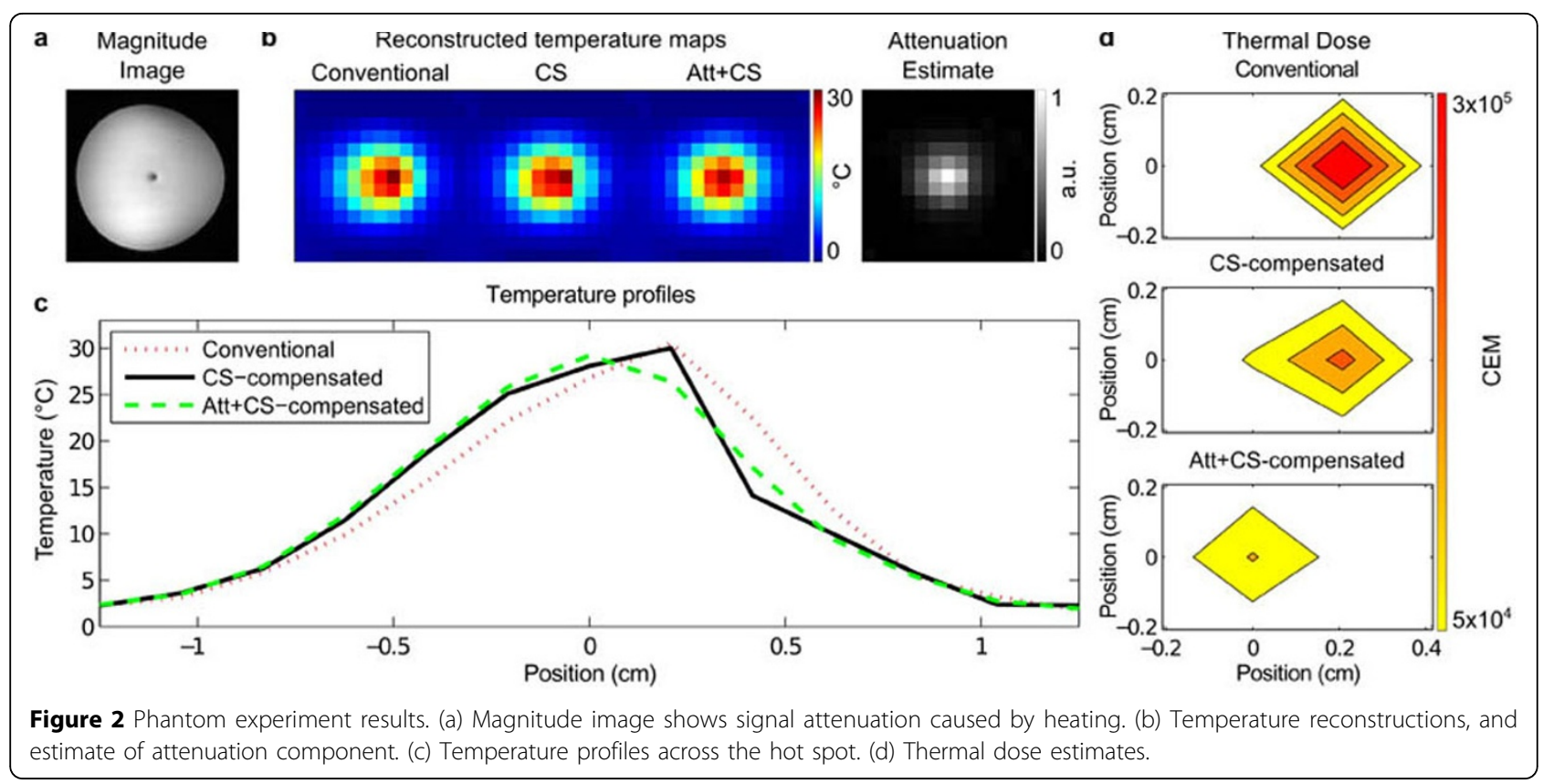

large dynamic scan acceleration factors[1] and the correction of chemical-shift (CS) effects that geometrically distort the temperature maps.[2] However, that work neglected image attenuation that accompanies the PRF phase shift and is primarily caused by increasing $\mathrm{T} 1$ with temperature.[3] Here it is shown that attenuation degrades the accuracy of k-space-based reconstructions, but that it can be accounted for in the reconstructions.

\section{Methods}

Simulations and experiments were performed using gradient-recalled echo scans at 3 Tesla (Philips Achieva with Sonalleve HIFU) with $16 \mathrm{~ms}$ echo time and $44 \mathrm{~Hz}$ bandwidth. A phantom was simulated with Gaussian-shaped hot spots ranging from 0 to pi and exponential attenuation factors ranging from 0 to 0.8 . A tissue-mimicking gel phantom was imaged and sonicated for $41 \mathrm{~s}$ with a $4 \mathrm{~mm}$ diameter treatment cell at $110 \mathrm{~W}$ and $1.2 \mathrm{MHz}$. Temperature maps were reconstructed using image-domain hybrid,[4] CS-compensated, $[2]$ and proposed joint attenuation- and CS-compensated approaches. The latter reconstructions were implemented as a refinement stage after a hybrid reconstruction. Gradient descent was used to iteratively update the temperature phase shift and exponential attenuation maps to minimize the error between the measured $\mathrm{k}$-space data and the treatment $\mathrm{k}$-space signal model.

\section{Results and conclusions}

Figure 1 shows the simulation results, which demonstrate that temperature reconstructions that do not account for image magnitude attenuation only partially correct chemical shift-induced geometric distortions.
Figure 2 shows the phantom experiment results, in which chemical shift distortions are more completely corrected when image magnitude attenuation is accounted for, and that the small reduction of error in the temperature map for each dynamic translates into a large difference in cumulative thermal dose. These results demonstrate that accounting for image magnitude attenuations improves $k$-space-based temperature map reconstructions, and that it is possible to jointly estimate PRF-shift temperature maps and accompanying image magnitude attenuations using an extended $\mathrm{k}$-space-based temperature reconstruction algorithm.

\section{Acknowledgements (Funding)}

This work was supported by the Focused Ultrasound Foundation, the HHMI/ VUMC Certificate Program in Molecular Medicine, NIH R25CA136440, and DoD W81XWH-12-BCRP-IDEA

Published: 30 June 2015

\section{References}

1. Gaur P, Grissom W: Accelerated MRI Thermometry by Direct Estimation of Temperature from Undersampled k-Space Data. Magn Reson Med 2014, In Press.

2. Gaur P, Grissom W: Temperature map reconstruction directly from kspace with compensation for heating-induced geometric distortions. Proc 22nd ISMRM 2014, 2362.

3. Rieke V, Pauly Butts K: MR Thermometry. J Magn Reson Imaging 2008, 27(2):376-390.

4. Grissom W, et al: Hybrid referenceless and multibaseline subtraction MR thermometry for monitoring thermal therapies in moving organs. Med Phys 2010, 37(9):5014-26.

doi:10.1186/2050-5736-3-S1-P19

Cite this article as: Gaur and Grissom: Improved k-space-based MR thermometry by joint PRF phase shift and $\mathrm{T} 1 / \mathrm{T} 2^{*}$ attenuation estimation. Journal of Therapeutic Ultrasound 2015 3(Suppl 1):P19. 